\title{
Resilience-performance trade-offs in managing social-ecological systems
}

\author{
Mehran Homavounfar $^{1}, \underline{\text { Rachata Muneepeerakul }}^{1}$ and $\underline{\text { John M. Anderies }}^{2}$
}

\begin{abstract}
Resilience-based approaches have been attracting attention in governing social-ecological systems facing rapid social and environmental changes. In this article, we investigate the governance policies that focus on resilience. Our analysis is built on a stylized dynamical model that mathematically operationalizes a widely used conceptual framework, which links social components, natural resources, and infrastructure in social-ecological systems. Specifically, we numerically solve the Hamilton-Jacobi-Bellman (HJB) equation to determine policies - in the form of investment in public infrastructure - that maximize a quantitative metric of the system's resilience. For comparison purposes, we also derive policies that maximize the system's performance and discuss the differences between and implications of the two policies. The results showed that a policy that maximizes performance results in sub-optimal resilience and vice versa. Moreover, our sensitivity analysis suggests that managing resilience requires that one be more responsive to changes in external forcing.
\end{abstract}

Key Words: dynamic optimization; ecological resilience; Hamilton-Jacobi-Bellman (HJB); resilience-based management; socialecological systems (SESs)

\section{INTRODUCTION}

Conventionally, approaches to resource allocation and environmental management focus on performance (Brouwer et al. 2002, Ganji et al. 2007, Homayoun-far et al. 2010, Anghinolfi et al. 2013, Önal et al. 2016, Little et al. 2017, Moradi and Rasaei 2017). These approaches focus on a few performance metrics of a system and search for policies that maximize expected utility or minimize expected cost. Although these command-and-control approaches may serve well in the early development phase of the resource use process, they are not well-equipped to address the complex, nonlinear interactions between system components, which determine the system's response to external shocks (Anderies et al. 2006). As such, they can inadvertently increase the vulnerability of the system against other disturbances (Csete and Doyle 2002).

As an alternative, resilience-based approaches, which are based on system thinking, are receiving more attention in the study of social-ecological systems (SESs). These approaches focus on maintaining the capacity of a system to cope with unexpected occurrences without changing from a desirable to an undesirable configuration. This is challenging because SESs often exhibit hysteresis and irreversible changes (Walker et al. 2002). Walker and Meyers (2004) noted that research on thresholds and evaluation of hysteresis effects is a priority topic in the area of sustainability science. Martin (2011) further explored the relationship between the idea of ecological resiliencespecifically in the case where a shock displaces a system beyond its elasticity threshold (see Carpenter et al. 2005) — and hysteresis. He combined the notion of resilience as used in ecology with hysteresis to examine the effects of a recession on an economic system. Lyver et al. (2019) investigated hysteresis in environmental management by evaluating the effects of environmental, social, and economic drivers on the resilience of traditional knowledge systems. They argued that breaking connections between people and their traditional lands and environment may move cultural and ecological systems toward a critical threshold. In addition, decisions for managing SESs must be made based on imperfect information (Walker et al. 2002). For example, distributions of a system's variables can be highly uncertain, i.e., deep uncertainty, or the parameters of the distributions may change faster than we can update information and, therefore, improper priors dominate the analysis. Incorporating these features makes resilience-based approaches better suited for managing SESs but also particularly challenging to analyze.

In the sustainability literature, many studies have discussed the resilience-based approaches in the management of SESs (Pezzey 1997, Fischer et al. 2009, Mäler and Li 2010, Derissen et al. 2011, Barfuss et al. 2018, Krueger et al. 2020). Fischer et al. (2009:549) proposed that "embedding optimization analyses within a resilience thinking framework could draw on the complementary strengths of the two bodies of work, thereby promoting costeffective and enduring conservation outcomes." Complicating the matter is the fact that promoting resilience can come at the expense of other desirable properties. Because of the existence of multiple thresholds and regime shifts, there is no perfect set of policies for governing SESs that maximizes resilience and performance simultaneously (Janssen and Anderies 2007, Ostrom et al. 2007, Homayounfar et al. 2018). Oftentimes, making a system secure against one source of disturbance increases vulnerability of the system against another (Janssen and Anderies 2007). According to Anderies et al. (2006), resilience-based approaches are effective in management of social-ecological systems not by providing a mechanism that can be used to predict the impact of management actions but, rather, by focusing on particular system attributes that play important roles in the dynamics of SESs and attempting to develop principles to guide interventions in SESs to improve their long-term resilience. In sum, resilience-based approaches focus on key controlling variables, alternate system regimes, and thresholds, whereas performance-based approaches focus on controlling natural variability and keeping the system in some perceived optimal state.

Although the literature on resilience is enormous and growing, so is cynicism about what the term means (much like the terms

\footnotetext{
${ }^{1}$ Agricultural and Biological Engineering Department, University of Florida, ${ }^{2}$ School of Human Evolution and Social Change and School of Sustainability, Arizona State University
} 
tipping point and sustainability), and there is a glaring lack of rigorous development of analytical tools to actually deploy resilience in practice. Anderies et al. (2013) explored these various ideas in qualitative terms and called for more rigorous analysis of trade-offs in managing resilience, robustness, and performance of SESs. In theory, these trade-offs are ubiquitous in SESs. However, to apply these analyses in real coupled systems, we need a deeper and more precise understanding of such trade-offs. This article is a step in that direction.

To investigate issues surrounding resilience-based management of SESs more rigorously, a reasonable, quantitative metric of resilience is needed. To this end, Walker et al. (2002) introduced a procedure to uncover resilient development pathways by combining adaptive management approaches (Holling 1978, Walters 1986) and scenario planning (Van der Heijden 1996). Anderies et al. (2004) further proposed a conceptual framework to assess robustness of SESs, from which Muneepeerakul and Anderies (2017) developed a stylized dynamical model. The model allows for mathematical definitions of the boundaries of policy domains that result in a sustainable system in which both human-made and natural infrastructure can be maintained over the long run. In this study, we investigated how to manage for resilience of the SES or, more generally, the coupled infrastructure system (CIS) represented by this model.

Regardless of whether the focus is on performance or resilience, decision makers are faced with the same challenges of appropriately taking into account the dynamics of natural and human-made components in response to implemented policies and disturbances. This is where dynamic programming is of value. These techniques have been used in natural resources management and bioeconomic models (Ganji et al. 2008, Homayounfar et al. 2011, Alvarez et al. 2016, Yaegashi et al. 2017, Zhao et al. 2017, Liu et al. 2018, Anderies et al. 2019).

In this article, we seek to formulate the problem of trade-offs between resilience and performance in coupled infrastructure systems in such a way that powerful analytical techniques of dynamic programming can be used to address the problem in a quantitative and systematic way. In particular, we developed Hamilton-Jacobi-Bellman (HJB) equations for a simplified version of the CIS model with an emphasis on infrastructure. In previous works, infrastructure was one of the dynamical variables in a higher-dimensional dynamical system but, because of the model complexity, the metric of resilience had to be determined numerically (e.g., Yu et al. 2015, Muneepeerakul and Anderies 2017, Homayounfar et al. 2018, Muneepeerakul and Anderies 2020), making it not very amenable to certain types of analytic tools. Here, through the simplification of the dynamical system model-making infrastructure the main dynamic variable although still embedding the effects of other variables - and the analytical expression of the system resilience, we were able to rigorously apply dynamical programming tools, namely the HJB approach. This allows for rigorous and systematic comparison between different aspects of the coupled system (e.g., resilience and performance). This rigorous approach was not possible in the previous works but was made possible here. We then compared the derived policies and conducted sensitivity analysis of these policies to changes in social and environmental settings. The results revealed the differences - and therefore trade-offs - in policies developed from these two philosophies.

\section{METHOD}

We analyzed the CIS model developed by Muneepeerakul and Anderies (2017), which captures dynamical behavior of the state of the public infrastructure, $I_{\mathrm{HM}}$, resource level, $R$, and the fraction of efforts resource users (RUs) spend inside the system, $U$ :

$$
\begin{gathered}
\frac{d R}{d t}=g-l R-U N R H\left(I_{\mathrm{HM}}\right) \\
\frac{d U}{d t}=r U(1-U)\left(\pi_{\mathrm{U}}-w\right) \\
\frac{d I_{\mathrm{HM}}}{d t}=\mu y p C U N R H\left(I_{\mathrm{HM}}\right)-\delta H\left(I_{\mathrm{HM}}\right)
\end{gathered}
$$

where $g$ is the replenishment rate of the resource, $l$ is the natural loss rate of the resource, $\delta$ is depreciation rate of infrastructure, $w$ is per capita revenue from working outside, $r$ is responsiveness of social actors to payoff differences, $\mu$ is maintenance effectiveness of public infrastructure providers' (PIPs) investment, $N$ is number of users, and $p$ is the conversion factor from resource to revenue. The function $H\left(I_{\mathrm{HM}}\right)$ maps $I_{\mathrm{HM}}$ to the productivity of each RU. $C$ and $y$ are policy parameters implemented by the PIPs: $C$ represents the fraction of their revenue that RUs contribute to PIPs for infrastructure maintenance, and $y$ is the fraction of all contributions received from the RUs that the PIPs invest in maintaining the infrastructure after compensating themselves. For further details about the above system, the reader is referred to Muneepeerakul and Anderies (2017).

Homayounfar et al. (2018) investigated this model for its resilience and robustness metrics. In that study, two types of specified resilience were considered: resilience against abandonment by PIPs and resilience against collapse of public infrastructure, which would be accompanied by abandonment by RUs. Each resilience was measured by how far the system was from the boundary of its respective mechanism of collapse (i.e., abandonment by PIPs or by RUs). Because loss of resilience of either type means the collapse of the system, the minimum between the two resilience metrics was taken as the resilience of the overall system. In Homayounfar et al. (2018), the resilience metric was numerically determined from stability conditions and, therefore, was not amenable to the present analysis. Therefore, we introduced the following quantity, which captures the qualitative behavior of the resilience matrix (determined numerically in Homayounfar et al. 2018), to capture the system resilience to be used in our analysis:

$$
\left\{\begin{array}{l}
\left(I_{\mathrm{HM}}-I_{\mathrm{c}}\right)\left(\pi_{\mathrm{p}}-w_{\mathrm{p}}\right), \text { when } I_{\mathrm{HM}}>I_{\mathrm{c}}, \pi_{\mathrm{p}}>w_{\mathrm{p}} \\
0, \quad \text { Otherwise }
\end{array}\right.
$$

where $I_{\mathrm{c}}$ indicates the critical state of the infrastructural services below which it is not possible to improve the infrastructure condition, $\pi_{\mathrm{p}}=(1-y) p C U N R H\left(I_{\mathrm{HM}}\right)$ is the net revenue that PIPs collect, and $w_{\mathrm{p}}$ is the opportunity cost that is earned if they choose to abandon this system and work with another system. Equation 2 exhibits zero resilience when the infrastructure is in too poor a 
Table 1. Definitions and interpretation of dimensionless groups. PIP = public infrastructure provider.

\begin{tabular}{lll}
\hline \hline Dimensionless group & Definition & Interpretation \\
\hline$x$ & $I_{\mathrm{HM}} / I_{\mathrm{m}}$ & Rescaled state of the public infrastructure \\
$\tau$ & $\delta t$ & Rescaled time \\
$x_{\mathrm{c}}$ & $I_{\mathrm{c}} / I_{\mathrm{m}}$ & $\begin{array}{l}\text { Rescaled critical state of infrastructure below which it is impossible to improve the } \\
\text { condition of the infrastructure }\end{array}$ \\
$\rho$ & $l / N h$ & Relative natural loss rate of the resource compared to the maximum harvest rate \\
$\vartheta_{\mathrm{m}}$ & $\delta I_{\mathrm{m}} / \mu p g$ & Decay rate of infrastructure at $I_{\mathrm{m}}$ relative to maximum maintenance \\
$\Phi_{1}$ & $\mathrm{pg} / \mathrm{wN}$ & Potential income- with the entire resource flow turned into income-relative to outside \\
& $\mathrm{pg} / \mathrm{w}_{\mathrm{P}}$ & wage \\
$\Phi_{2}$ & & Potential income relative to PIP's alternative opportunities \\
\hline
\end{tabular}

condition $\left(I_{\mathrm{HM}} \leq I_{\mathrm{c}}\right)$ or the PIPs' payoff is too low $\left(\pi_{\mathrm{p}} \leq w_{\mathrm{p}}\right)$ and exhibits higher values as the system is farther away from these zero-resilience boundaries. Simply speaking, positive values of $\left(I_{\mathrm{HM}}-I_{\mathrm{c}}\right)\left(\pi_{\mathrm{p}}-w_{\mathrm{p}}\right)$ indicate that the system is resilient against being abandoned by PIPs and can absorb shocks to the infrastructure although maintaining livelihoods of the RUs. This is the same qualitative behavior observed in Homayounfar et al. (2018). The quantity in Equation 2 captures the capacity of the system to produce enough economic value to maintain both those who rely directly on the resource and cover the costs of governing shared resources (the income of the PIPs).

To simplify the analysis, we made a timescale separation argument based on the assumption that the dynamics of $U$, the fraction of efforts RUs spend inside the system (days or weeks), and $R$, resource level (weeks or months), were significantly faster than $I_{\mathrm{HM}}$, the state of the public infrastructure (years or decades), because establishment and development of human-made infrastructures, either soft or hard, is a time-consuming process (see Appendix 1 for more details). In this case, we set $d R / d t=0$ and $d U / d t=0$ - focusing only on the non-trivial, interior solution of $U$ - and derived $R$ and $U$ as functions of $I_{\mathrm{HM}}$ and rewrote the dynamic of $I_{\mathrm{HM}}$ as follows:

$$
\frac{d I_{\mathrm{HM}}}{d t}=\mu y p C\left(g-\frac{l w}{(1-C) p H\left(I_{\mathrm{HM}}\right)}\right)-\delta H\left(I_{\mathrm{HM}}\right)
$$

In sum, the dynamics of the CIS was now summarized by the dynamics of public infrastructure on which our focus was now placed. Non-dimensionalizing Equation 3 and using the resulting dimensionless groups, the equation for human-made infrastructure became:

$$
\frac{d x}{d \tau}=\frac{y C}{\theta_{\mathrm{m}}}\left(1-\frac{\rho}{(1-C) \varnothing_{1} H(x)}\right)-x
$$

The resilience of the system was then rewritten as:

$$
\left\{\begin{array}{l}
\left(x-x_{\mathrm{c}}\right)\left(\pi_{\mathrm{p}} / w_{\mathrm{p}}-1\right), \text { when } x>x_{\mathrm{c}}, \pi_{\mathrm{p}}>w_{\mathrm{p}} \\
0, \quad \text { Otherwise }
\end{array}\right.
$$

where $x=I_{\mathrm{HM}} / I_{\mathrm{m}} \mathrm{x}_{\mathrm{c}}=I_{\mathrm{c}} / I_{\mathrm{m}}$ and $\tau=\delta t$ are dimensionless groups associated with $I_{\mathrm{HM}}, I_{\mathrm{c}}$, and $t$, respectively (Table 1 ).

The term $\pi_{\mathrm{p}} / w_{\mathrm{p}}$ represents the ratio between the payoff for the PIPs to the alternative payoff if they decide to manage another system and can be written as follows (see Table 1 for definitions and interpretation of the dimensionless groups):

$$
\frac{\pi_{\mathrm{p}}}{w_{\mathrm{p}}}=(1-y) C \varnothing_{2}\left(1-\frac{\rho}{(1-C) \emptyset_{1} H(x)}\right)
$$

Putting these elements together, our problem to maximize resilience can be written as:

$$
\begin{gathered}
\operatorname{Max}_{y} \int_{0}^{\infty} e^{\frac{\rho^{\prime}}{\delta} \tau}\left(x-x_{\mathrm{c}}\right)\left(\pi_{\mathrm{p}} / w_{\mathrm{p}}-1\right) d \tau \\
\text { Subject to: } \frac{d x}{d \tau}=\frac{y C}{\theta_{\mathrm{m}}}\left(1-\frac{\rho}{(1-C) \varnothing_{1} H(x)}\right)-x
\end{gathered}
$$

Where $\rho^{\prime}$ is the discount rate, not to be confused with $\rho$, a dimensionless group in the dynamical equation. We will use $\hat{y}_{R}$ to denote the solution to the above problem, which is the investment policy that maximizes the system resilience.

The HJB equation corresponding to the problem presented by Equation 7 is:

$$
\frac{\rho^{\prime}}{\delta} V(x)=\operatorname{Max}_{y}\left[\left(x-x_{\mathrm{c}}\right)\left(\pi_{\mathrm{p}} / w_{\mathrm{p}}-1\right)+\frac{d V}{d x} \frac{d x}{d \tau}\right]
$$

where $V$ is the so-called "value function" representing the integral in Equation 7 associated with the optimal policy, $\hat{y}_{R}$, which we will derive later.

To make the comparison with performance-based approaches, we defined two objective functions $\pi_{\mathrm{p}}$ and $U N w+\pi_{\mathrm{p}}$ representing, respectively, the PIP performance and the system performance, i.e., revenue, of the system: $U N w$ is the overall payoff to the resource users, and $\pi_{\mathrm{p}}$, as before, is the payoff to the PIPs. From 
the mathematical definition, the former function represents a selfish social planner who does not care about the users, whereas the latter reflects a benevolent social planner looking after all social entities involved in the system. These functions were nondimensionalized into $\pi_{\mathrm{p}} / w_{\mathrm{p}}$ and $U+\pi_{\mathrm{p}} / w N$. Note that, because 1$U$ can be thought of as a metric of out-migration from the system, the results associated with the function $U+\pi_{\mathrm{p}} / w N$ have implications on the system migration process. Through the same procedure, we arrived at the following corresponding HJB equations for the performance-based governance:

$$
\begin{gathered}
\frac{\rho^{\prime}}{\delta} V(x)=\underset{y}{\operatorname{Max}}\left[U+\frac{\pi_{\mathrm{p}}}{N w}+\frac{d V}{d x} \frac{d x}{d \tau}\right] \\
\frac{\rho^{\prime}}{\delta} V(x)=\underset{y}{\operatorname{Max}}\left[\frac{\pi_{\mathrm{p}}}{w_{\mathrm{p}}}+\frac{d V}{d x} \frac{d x}{d \tau}\right]
\end{gathered}
$$

We will use $\hat{y}_{\text {P-PIP }}$ and $\hat{y}_{\text {P-SYS }}$ to denote the investment policies that maximize the PIP performance and the system performance, respectively.

Finally, we also conducted a simple sensitivity analysis on how sensitive these optimal policies were to changes in socialecological settings. In particular, we simply varied two parameters in the original model (Eq. 1): the per capita wage of working outside the system $(w$, social) and the resource replenishment rate ( $g$, ecological), and considered how $\hat{y}_{R}, \hat{y}_{\text {P-PIP }}$ and $\hat{y}_{\text {P-SYS }}$ varied with them.

\section{RESULTS AND DISCUSSION}

We first established $x_{c}$ as the critical level of infrastructure below which it is impossible to improve the condition of the infrastructure. In other words, at this level, it is not worthwhile to invest in infrastructure maintenance. We derived $x_{\mathrm{c}}$ from the condition $d x /\left.d \tau\right|_{y}=1 \leq 0$, that is, $x$ cannot improve despite the PIPs investing everything they have collected from the resource users (RUs). This leads to the following expression of $x_{\mathrm{c}}$ :

$$
\frac{1}{2}\left[x_{0}+\frac{C}{\theta_{\mathrm{m}}}-\sqrt{\left(x_{0}+\frac{C}{\theta_{\mathrm{m}}}\right)^{2}-\frac{4 C}{\theta_{\mathrm{m}}}\left[x_{0}+\rho \frac{1-x_{0}}{(1-C) \emptyset_{1}}\right]}\right]
$$

We then analyzed the HJB equations (Eqs. 8, 9, and 10), which yielded the following optimal policies:

$$
\hat{y}_{\mathrm{R}}=\left\{\begin{array}{l}
1,\left[\frac{d V}{d x} \frac{C}{\theta_{\mathrm{m}}}-C \varnothing_{2}\left(x-x_{\mathrm{c}}\right)\right]\left(1-\frac{\rho}{(1-C) \emptyset_{1} H(x)}\right)>0 \\
0, \quad \text { Otherwise }
\end{array}\right.
$$

$$
\begin{aligned}
& \hat{y}_{\mathrm{P}-\mathrm{PIP}}=\left\{\begin{array}{l}
1,\left[\frac{d V}{d x} \frac{C}{\theta_{\mathrm{m}}}-C \varnothing_{2}\right]\left(1-\frac{\rho}{(1-C) \emptyset_{1} H(x)}\right)>0 \\
0,
\end{array}\right. \\
& \hat{y}_{\mathrm{P}-\mathrm{SYS}}=\left\{\begin{array}{l}
1,\left[\frac{d V}{d x} \frac{C}{\theta_{\mathrm{m}}}-C \varnothing_{1}\right]\left(1-\frac{\rho}{(1-C) \emptyset_{1} H(x)}\right)>0 \\
0, \quad \text { Otherwise }
\end{array}\right.
\end{aligned}
$$

In all three cases, it turned out that the optimal policies are the so-called bang-bang control: investing either everything $(y=1)$ or nothing $(y=0)$ depending on with the state of the infrastructure. In particular, under these optimal policies, the PIPs invest everything when $x_{\mathrm{c}}<x<x_{1}$ and nothing when $x_{\mathrm{c}}>x$ or $x>x_{1}$ (Fig. 1); $x_{1}$ was numerically determined from the conditions under which $\hat{y}_{R}, \hat{y}_{\text {P-PIP }}$ or $\hat{y}_{\text {P-SYS }}$ is equal 1. Bang-bang control strategies have been found in linear and nonlinear optimal control problems involving infrastructure systems (e.g., Friesz et al. 1979, Cave and Vogelsang 2003, Zandvliet et al. 2007, Hritonenko and Yatsenko 2010, Dahlgren and Leung 2015, Bolzoni et al. 2019).

Fig. 1. (A) The schematic illustration of the relationship between an optimal policy for investing in the public infrastructure, $y$, and the state of public infrastructure, $x$. Here, $x_{\mathrm{c}}$ represents the critical state of the infrastructural services below which it is not possible to improve the infrastructure condition, and $x_{1}$ indicates the state beyond which the investment stops. (B) $H(x)$ is the function that maps $x$ to the productivity of each RU. Here, $x_{0}$ is the threshold of $x$ below which productivity function, $H$, is zero.

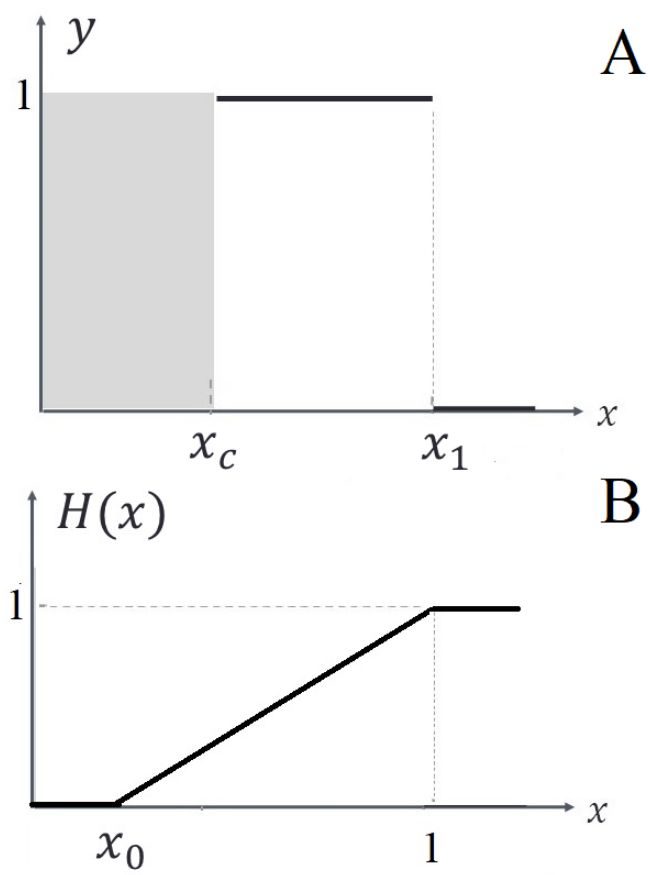


Generally, a complete analytical solution to optimization problems such as ours is difficult (Boscain and Piccoli 2005). Here, we solved the HJB equations numerically to obtain the value function $V$ (Fig. 2). Functions $V$ exhibit bigjumps at $x_{\mathrm{c}}$. The figure also illustrates that, for the initial states lower than $x_{c}$, there is no reason for making an investment in the infrastructure: with its infrastructure in such a poor condition, the RUs cannot generate enough revenue to pass on to the PIPs to maintain the infrastructure and, consequently, the system is doomed to collapse in the long run. This resonates with many human-made infrastructures such as local irrigation canal systems, flood control, and water transfer systems, where infrastructural services (either soft or hard) play a key role in survival of those systems.

Fig. 2. The relationship between the value functions, $V$, and the initial value of $x$, state of public infrastructure. (A) Resiliencebased approach, (B) performance-based approach considering the system performance, and (C) performance-based approach considering the PIP performance. PIP = public infrastructure provider.
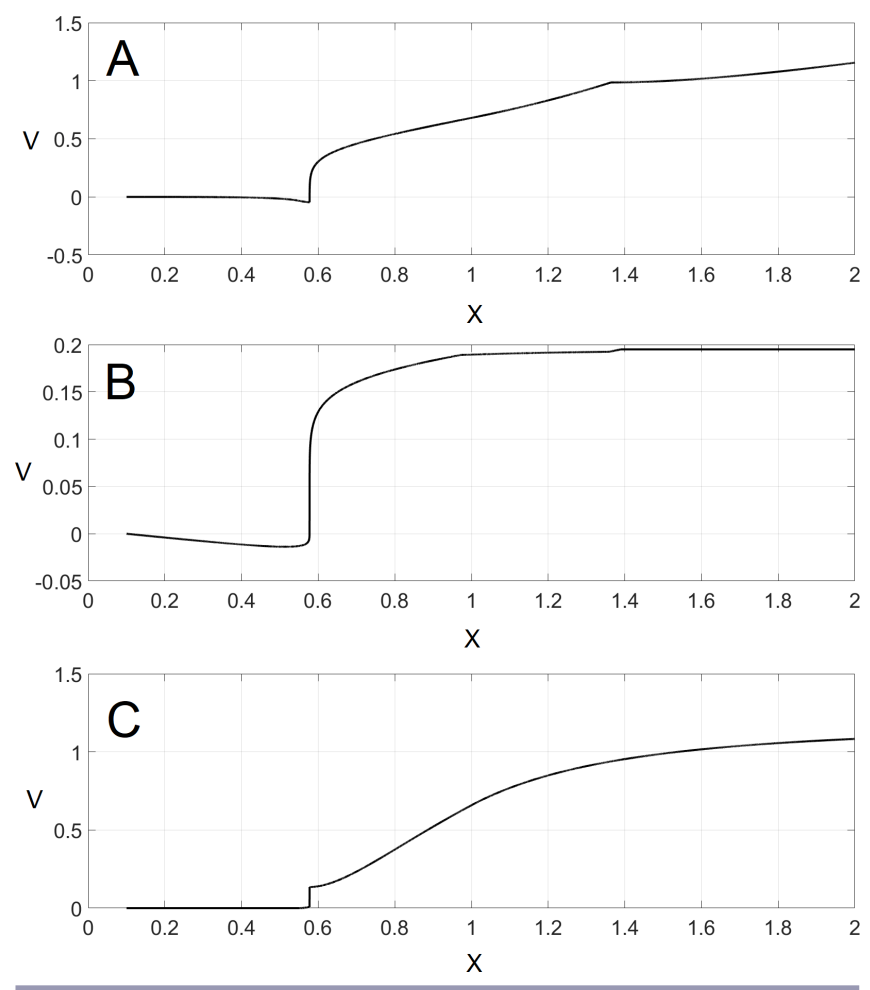

The differences between $\hat{y}_{\mathrm{R}}, \hat{\mathrm{y}}_{\mathrm{P}-\mathrm{PIP}}$, and $\hat{\mathrm{y}}_{\mathrm{P}-\mathrm{SYS}}$ are the level of $x$ at which the maintenance investment stops, i.e., $x_{1, \mathrm{R}}$ vs. $x_{1, \mathrm{P}-\mathrm{PIP}}$ and $x_{1 \text { P-SYS }}$, and how they vary with the taxation level $C$. The objective of $\hat{y}_{\text {P-SYS }}$ is to maximize overall performance, $U+\pi_{\mathrm{p}} / N w$, in the long run, so any investment for other purposes is considered wasteful. In this analysis, the relationship $H(x)$ between infrastructure condition and productivity of each RU (Fig. 1) is such that there is no gain in productivity beyond $x=1$ (many shared infrastructures exhibit similar nonlinear behavior in their productivity). This explains why $x_{1, \mathrm{P}-\mathrm{PIP}}$ and $x_{1, \mathrm{P}-\mathrm{SYS}} \leq 1$.

Whereas $x_{1, \mathrm{P}-\mathrm{SYS}}$ is equal to 1 over all values of $C, x_{1, \mathrm{P}-\mathrm{PIP}}$ increases monotonically with $C$ until a certain point, after which it levels off at 1 (Fig. 3). The difference between $x_{1, \text { P-PIP }}$ and $x_{1, \mathrm{P}-\mathrm{SYS}}$ highlights the difference in the social planner's objectives. A benevolent social planner who is concerned about the performance of both PIPs and RUs aims to maximize the overall system's performance, resulting in more investment infrastructure than that seen in case where the sole focus is on the PIPs' performance. For the resilience-based approach, $x_{1, \mathrm{R}}$ exhibits a hump-shaped relationship with $C$, peaking at an intermediate $C$. Importantly, $x_{1, \mathrm{R}}$ is always greater than $x_{1, \mathrm{P}-\mathrm{PIP}}$ and $x_{1, \mathrm{P}-\mathrm{SYS}}$. Maintaining the infrastructure in a better condition enables the system to absorb greater infrastructural shocks-greater resilience.

Fig. 3. $x_{c}$ is the critical level of infrastructure below which it is impossible to improve the condition of the infrastructure. $x_{1, \mathrm{R}}$, $x_{1, \mathrm{P}-\mathrm{PIP}}, x_{1, \mathrm{P}-\mathrm{SYS}}$ indicate the level of $x$ at which the maintenance investment stops and are associated with resilience-based and performance-based approaches under various $C$ 's and in different scenarios defined by the replenishing rate of the resource, $g$, and per capita revenue from working outside, $w: g$ $=(90,100,110)$ and per capita wage from working outside, $w=$ $(0.9,1,1.1)$.
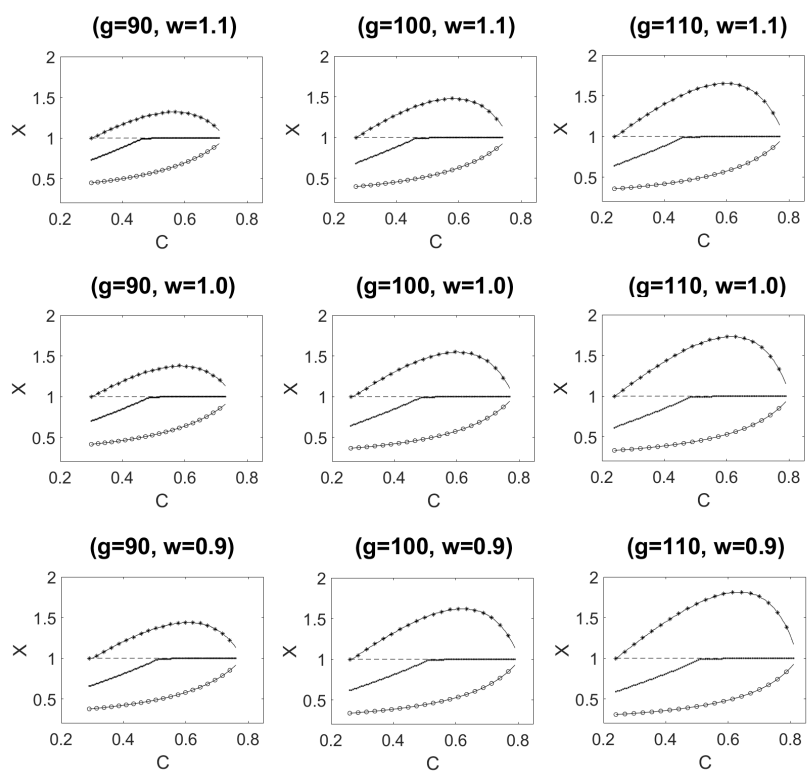

$$
x_{\mathrm{c}} \rightarrow \hat{x}_{1, \mathrm{R}} \rightarrow \quad, \hat{x}_{1, \mathrm{P}-\mathrm{SYS}} \quad-\quad, \hat{x}_{1, \mathrm{P}-\mathrm{PIP}} \quad \ldots . .
$$

We also conducted a sensitivity analysis of these optimal policies to changes in social-ecological settings, namely changes in replenishing rate of resource $g$ and per capita wage from working outside $w$ (Fig. 3). When the system enjoys great resource availability (high $g$ ) and incentive for the RUs to leave the system is low (e.g., Fig. 3: $g=110$ and $w=0.9$ ), $x_{1, \mathrm{R}}$ increases, and vice versa, when $g$ is low and $w$ is high (e.g., Fig. 3: $g=90$ and $w=$ $1.1)$. In contrast, $x_{1, \mathrm{P}-\mathrm{PIP}}$ and $x_{1, \mathrm{P}-\mathrm{SYS}}$ are confined to 1 in all socialecological settings, exhibiting much less sensitivity to changes in $g$ and $w$. This pointed to a more nuanced trade-off between policy resilience and system resilience. This is in keeping with the "resilience of what to what" notion in the resilience literature. 
Fig. 4. An illustration of the tension and trade-off between resilience-based and performance-based approaches in managing social-ecological systems.

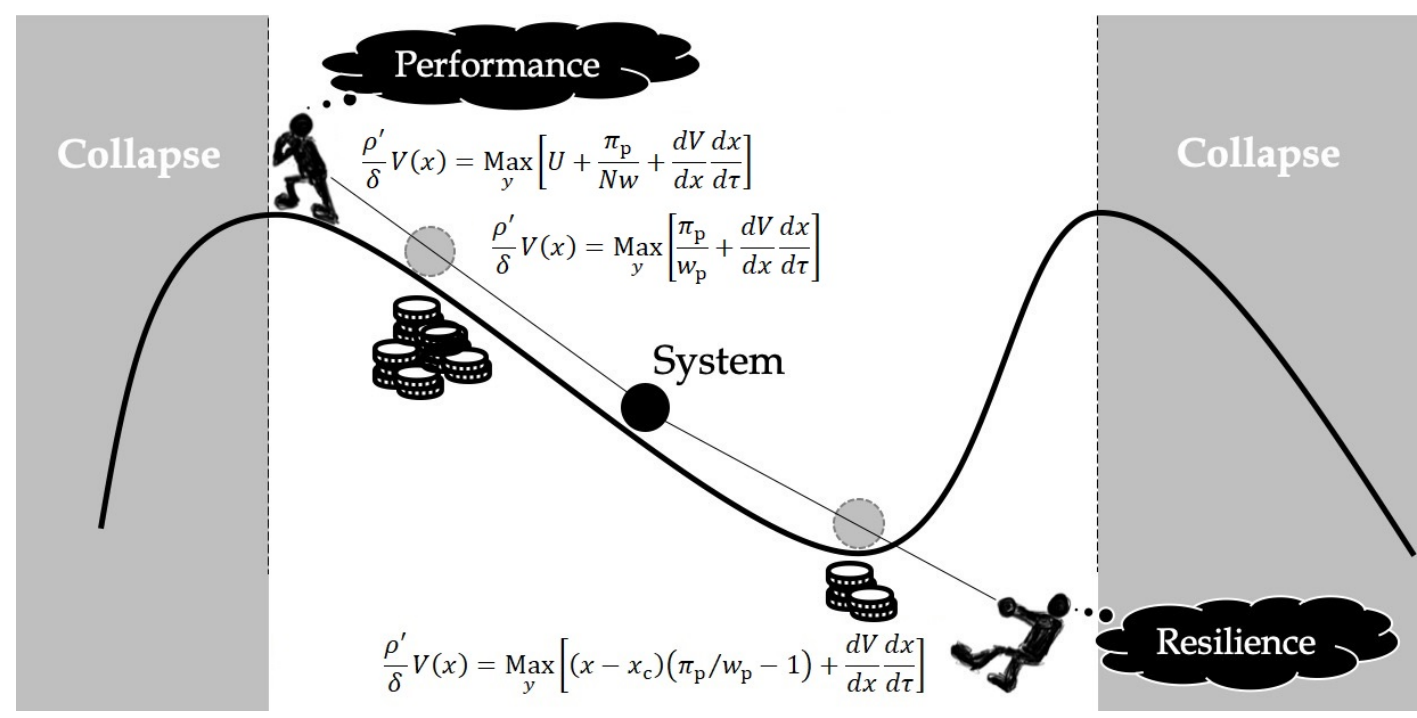

What our analysis focused on was the resilience of the coupled system, not the resilience of the policy. The policy for the less resilient outcomes (e.g., those associated with maximum PIPs' performance) is less sensitive to external factors (and may be seen as more resilient). On the other hand, for the system to be more resilient, the policy to achieve that must be more adaptive to external factors (and can be seen as less resilient), an important implication for policy makers.

Finally, it should be noted that our resilience objective function (Eq. 6) is related to the ecological resilience as defined by C. S. Holling and colleagues (Holling 1973, Walker et al. 2004), which can be linked to the size of basin of attraction and/or the distance to the boundary of another regime - concepts/quantities that can be defined in deterministic dynamical systems. In particular, a small value of the resilience objective function means that the system may sustain without perturbation but will quickly collapse if exposed to a small perturbation. If the social planner designs a policy under an (unrealistic) assumption that there would be no perturbation, she would prioritize the conventional performance objective function; her system may persist for a while, but will collapse when even a small perturbation occurs. This underlines the importance of the type of resilience-focused analysis conducted here. Building on this work, adding stochastic shocks to the system will enrich the analysis and the notion of resilience (see, e.g., Drury and Lodge 2009) and will be a worthwhile future research direction.

\section{CONCLUSION}

In this study, we applied dynamic programming to a dynamical model of social-ecological systems (SESs) or, more generally, coupled infrastructure systems (CISs), that places emphasis on the roles of infrastructure (Anderies et al. 2004, Muneepeerakul and Anderies, 2017). In particular, we derived the HJB equations based on a simplified dynamics of public infrastructure and determined the investment policies for infrastructure maintenance that maximize, respectively, system resilience, system performance, and PIP performance, namely $\hat{y}_{R}, \hat{y}_{\text {P-SYS }}$, and $\hat{\mathrm{y}}_{\mathrm{P} \text { PIP }}$, and showed that they differed. $\hat{\mathrm{y}}_{\mathrm{R}}, \hat{\mathrm{y}}_{\mathrm{P}-\mathrm{SYS}}$ and $\hat{\mathrm{y}}_{\mathrm{P} \text {-PIP }}$ are optimal for their respective objectives, so the fact that they differ implies trade-offs between them: a policy that maximizes performance results in sub-optimal resilience and vice versa. Between the two performance objective functions, taking into consideration the payoff of resource users results in greater investment in infrastructure maintenance. These differences add to complexity and challenges in governing SESs: it is well-known that there is a trade-off between performance and robustness. Homayounfar et al. (2018) pointed out the trade-off between resilience and robustness, and now this study shows the trade-off between resilience and performance (Fig. 4) - many trade-offs for one to navigate in governing SESs. In addition, the sensitivity analysis showed that the investment policy that maximize resilience, i.e., $\hat{y}_{R}$, is more sensitive to changes in social-ecological settings than the investment policies that maximize performance, i.e., $\hat{y}_{\text {P-SYS }}$ and $\hat{y}_{\text {P-PIP }}$, implying a nuanced trade-off between system resilience and policy resilience - managing for resilience requires that one be more responsive to changes in external forcing.

Effective governance of SESs involves multiple dimensions and requires one's ability to navigate the complex trade-offs among them. The methodology employed here can be useful for such a task. We hope that studies such as ours would enable environmental scientists and professionals to determine how environmental policies and institutions should change in response to changes in social and environmental factors in a more holistic way.

Responses to this article can be read online at: https://www.ecologyandsociety.org/issues/responses. php/12892 


\section{Acknowledgments:}

MH and RM acknowledge support from the Army Research Officel Army Research Laboratory. This research was supported by the Army Research Officel Army Research Laboratory under award no. W911NF1810267 (Multidisciplinary University Research Initiative). The views and conclusions in this document are those of the authors and should not be interpreted as representing the official policies either expressed or implied of the Army Research Office or the United States Government.

\section{Data Availability:}

The authors provide all relevant data and code underlying the findings described in the manuscript.

\section{LITERATURE CITED}

Alvarez, M. E., S. Di Marco, K. Erdlenbruch, and M. Tidball. 2016. A mixed control problem of the management of natural resources. Natural Resource Modeling 29:353-373. https://doi. org/10.1111/nrm.12085

Anderies, J. M., C. Folke, B. Walker, and E. Ostrom. 2013. Aligning key concepts for global change policy: robustness, resilience, and sustainability. Ecology and Society 18(2):8. https:// doi.org/10.5751/ES-05178-180208

Anderies, J. M., M. A. Janssen, and E. Ostrom. 2004. A framework to analyze the robustness of social-ecological systems from an institutional perspective. Ecology and Society 9(1):18. https://doi. org/10.5751/ES-00610-090118

Anderies, J. M., J. D. Mathias, and M. A. Janssen. 2019. Knowledge infrastructure and safe operating spaces in socialecological systems. Proceedings of the National Academy of Sciences 116:5277-5284. https://doi.org/10.1073/pnas.1802885115

Anderies, J. M., B. H. Walker, and A. P. Kinzig. 2006. Fifteen weddings and a funeral: case studies and resilience-based management. Ecology and Society 11(1):21. https://doi. org/10.5751/ES-01690-110121

Anghinolfi, D., M. Paolucci, M. Robba, and A. C. Taramasso. 2013. A dynamic optimization model for solid waste recycling. Waste Management 33:287-296. https://doi.org/10.1016/j. wasman.2012.10.006

Barfuss, W., J. F. Donges, S. J. Lade, and J. Kurths. 2018. When optimization for governing human-environment tipping elements is neither sustainable nor safe. Nature Communications 9(1):1-10. https://doi.org/10.1038/s41467-018-04738-Z

Bolzoni, L., E. Bonacini, R. Della Marca, and M. Groppi, 2019. Optimal control of epidemic size and duration with limited resources. Mathematical Biosciences 315:108232. https://doi. org/10.1016/j.mbs.2019.108232

Boscain, U., and B. Piccoli. 2005. An introduction to optimal control. Pages 19-66. Contrôle non linéaire et applications. Herman, Paris, France.
Brouwer, D. R., and J. D. Jansen. 2002. Dynamic optimization of waterflooding with smart wells using optimal control theory. Society of Petroleum Engineers 9(4):391-402. https://doi. org/10.2118/78278-MS

Carpenter, S. R., F. Westley, and M. G. Turner. 2005. Surrogates for resilience of social-ecological systems. Ecosystems 8:941-944. https://doi.org/10.1007/s10021-005-0170-y

Cave, M., and I. Vogelsang. 2003. How access pricing and entry interact. Telecommunications Policy 27:717-727. https://doi. org/10.1016/j.telpol.2003.08.004

Csete, M. E., and J. C. Doyle. 2002. Reverse engineering of biological complexity. Science 295:1664-1669. https://doi. org/10.1126/science.1069981

Dahlgren, E., and T. Leung. 2015. An optimal multiple stopping approach to infrastructure investment decisions. Journal of Economic Dynamics and Control 53:251-267. https://doi. org/10.1016/j.jedc.2015.02.001

Derissen, S., M. F. Quaas, and S. Baumgärtner. 2011. The relationship between resilience and sustainability of ecologicaleconomic systems. Ecological Economics 70(6):1121-1128. https://doi.org/10.1016/j.ecolecon.2011.01.003

Drury, K. L. S., and D. M. Lodge. 2009. Using mean first passage times to quantify equilibrium resilience in perturbed intraguild predation systems. Theoretical Ecology 2(1):41-51. https://doi. org/10.1007/s12080-008-0027-Z

Fischer, J., G. D. Peterson, T. A. Gardner, L. J. Gordon, I. Fazey, T. Elmqvist, A. Felton, C. Folke, and S. Dovers. 2009. Integrating resilience thinking and optimisation for conservation. Trends in Ecology \& Evolution 24(10):549-554. https://doi.org/10.1016/j. tree.2009.03.020

Friesz, T. L., and J. E. Fernandez. 1979. A model of optimal transport maintenance with demand responsiveness. Transportation Research Part B: Methodological 13(4):317-339. https://doi. org/10.1016/0191-2615(79)90025-0

Ganji, A., D. Khalili, and M. Karamouz. 2007. Development of stochastic dynamic nash game model for reservoir operation. I. The symmetric stochastic model with perfect information. Advances in Water Resources 30:528-542. https://doi. org/10.1016/j.advwatres.2006.04.004

Ganji, A., D. Khalili, M. Karamouz, K. Ponnambalam, and M. Javan. 2008. A fuzzy stochastic dynamic nash game analysis of policies for managing water allocation in a reservoir system. Water Resources Management 22:51-66. https://doi.org/10.1007/ s11269-006-9143-y

Holling, C. S. 1973. Resilience and stability of ecological systems. Annual Review of Ecology and Systematics 4:1-23. https://doi. org/10.1146/annurev.es.04.110173.000245

Holling, C. S. 1978. The spruce-budworm/forest-management problem. Pages 143-182 in C. S. Holling, editor. Adaptive environmental assessment and management. John Wiley, New York, New York, USA.

Homayoun-far, M., A. Ganji, D. Khalili, and J. Harris. 2010. Two solution methods for dynamic game in reservoir operation. 
Advances in Water Resources 33:752-761. https://doi. org/10.1016/j.advwatres.2010.04.001

Homayounfar, M., A. Ganji, and C. J. Martinez. 2011. A novel solution for stochastic dynamic game of water allocation from a reservoir using collocation method. Water Resources Management 25:3427-3444. https://doi.org/10.1007/s11269-011-9863-5

Homayounfar, M., R. Muneepeerakul, J. M. Anderies, and C. P. Muneepeerakul. 2018. Linking resilience and robustness and uncovering their trade-offs in coupled infrastructure systems. Earth System Dynamics 9:1159-1168. https://doi.org/10.5194/ esd-9-1159-2018

Hritonenko, N., and Y. Yatsenko. 2010. Age-structured PDEs in economics, ecology, and demography: optimal control and sustainability. Mathematical Population Studies 17(4):191-214. https://doi.org/10.1080/08898480.2010.514851

Janssen, M. A., and J. M. Anderies. 2007. Robustness trade-offs in social-ecological systems. International Journal of the Commons 1:43-66. https://doi.org/10.18352/ijc.12

Krueger, E. H., D. Borchardt, J. W. Jawitz, and P. S. C. Rao. 2020. Balancing security, resilience, and sustainability of urban water supply systems in a desirable operating space. Environmental Research Letters 15:035007. https://doi.org/10.1088/1748-9326/ ab6c2d

Little, K. W., N. H. Koralegedara, C. M. Northeim, and S. R. AlAbed. 2017. Decision support for environmental management of industrial non-hazardous secondary materials: new analytical methods combined with simulation and optimization modeling. Journal of Environmental Management 196:137-147. https://doi. org/10.1016/j.jenvman.2017.02.075

Liu, B., C. Cheng, S. Wang, S. Liao, K. W. Chau, X. Wu, and W. Li. 2018. Parallel chance-constrained dynamic programming for cascade hydropower system operation. Energy 165:752-767. https://doi.org/10.1016/j.energy.2018.09.140

Lyver, P. O’B., P. Timoti, T. Davis, and J. M. Tylianakis. 2019. Biocultural hysteresis inhibits adaptation to environmental change. Trends in Ecology \& Evolution 34(9):771-780. https://doi. org/10.1016/j.tree.2019.04.002

Mäler, K. G., and C. Z. Li. 2010. Measuring sustainability under regime shift uncertainty: a resilience pricing approach. Environment and Development Economics 15:707-719. https:// doi.org/10.1017/S1355770X10000318

Martin, R. 2011. Regional economic resilience, hysteresis and recessionary shocks. Journal of Economic Geography 12:1-32. https://doi.org/10.1093/jeg/1br019

Moradi, T., and M. R. Rasaei. 2017. Automated reservoir management using multi-objective simulation optimization and SA model. Journal of Petroleum Science and Engineering 150:91-98. https://doi.org/10.1016/j.petrol.2016.11.024

Muneepeerakul, R., and J. M. Anderies. 2017. Strategic behaviors and governance challenges in social-ecological systems. Earth's Future 5:865-876. https://doi.org/10.1002/2017EF000562

Muneepeerakul, R., and J. M. Anderies. 2020. The emergence and resilience of self-organized governance in coupled infrastructure systems. Proceedings of the National Academy of Sciences 117:4617-4622. https://doi.org/10.1073/pnas.1916169117

Önal, H., P. Woodford, S. A. Tweddale, J. D. Westervelt, M. Chen, S. T. M. Dissanayake, and G. Pitois. 2016. A dynamic simulation/ optimization model for scheduling restoration of degraded military training lands. Journal of Environmental Management 171:144-157. https://doi.org/10.1016/j.jenvman.2016.02.005

Ostrom, E., M. A. Janssen, and J. M. Anderies. 2007. Going beyond panaceas. Proceedings of the National Academy of Sciences 104:15176-15178. https://doi.org/10.1073/pnas.0701886104

Pezzey, J. C. V. 1997. Sustainability constraints versus "optimality" versus intertemporal concern, and axioms versus data. Land Economics 73:448-466. https://doi.org/10.2307/3147239

Van der Heijden, K. 1996. Scenarios: the art of strategic conversation. John Wiley, New York, New York, USA.

Walker, B., S. Carpenter, J. Anderies, N. Abel, G. S. Cumming, M. Janssen, L. Lebel, J. Norberg, G. D. Peterson, and R. Pritchard. 2002. Resilience management in social-ecological systems: a working hypothesis for a participatory approach. Conservation Ecology 6(1):14. https://doi.org/10.5751/ES-00356-060114

Walker, B., C. S. Holling, S. R. Carpenter, and A. Kinzig. 2004. Resilience, adaptability and transformability in social-ecological systems. Ecology and Society 9(2):5. https://doi.org/10.5751/ ES-00650-090205

Walker, B., and J. A. Meyers. 2004. Thresholds in ecological and social-ecological systems: a developing database. Ecology and Society 9(2):3. https://doi.org/10.5751/ES-00664-090203

Walters, C. J. 1986. Adaptive management of renewable resources. Macmillan, New York, New York, USA.

Yaegashi, Y., H. Yoshioka, K. Unami, and M. Fujihara. 2017. An optimal management strategy for stochastic population dynamics of released Plecoglossus altivelis in rivers. International Journal of Modeling, Simulation, and Scientific Computing 8:1750039. https://doi.org/10.1142/S1793962317500398

Yu, J. D., M. R. Qubbaj, R. Muneepeerakul, J. M. Anderies, and R. M. Aggarwal. 2015. Effect of infrastructure design on commons dilemmas in social-ecological system dynamics. Proceedings of the National Academy of Sciences 112:13207-13212. https://doi.org/10.1073/pnas.1410688112

Zandvliet, M. J., O. H. Bosgra, J. D. Jansen, P. M. J. Van den Hof, and J. F. B. M. Kraaijevanger. 2007. Bang-bang control and singular arcs in reservoir flooding. Journal of Petroleum Science and Engineering 58:186-200. https://doi.org/10.1016/j.petrol.2006.12.008

Zhao, T., J. Zhao, X. Lei, X. Wang, and B. Wu. 2017. Improved dynamic programming for reservoir flood control operation. Water Resources Management 31:2047-2063. https://doi. org/10.1007/s11269-017-1599-4 


\section{Appendix A. Appendix}

\section{Basic model}

Basic model. Here we briefly describe the basic model presented by Muneepeerakul and Anderies (2017). The model shows dynamic behaviour of three principal variables, namely, the state of the public infrastructure, $I_{H M}$, resource level, $R$, and the fraction of time user makes use of infrastructure, $U$, through Eqs. A.1. A.4. A.5. The schematic diagram of this system of equations is shown in Fig. A1.

In this context, $I_{H M}$ depends on PIPs in term of maintenance cost and has a positive relationship with the capacity of users to create resource flows. Eq. A.1 illustrates the dynamics of $I_{H M}$ as follows:

$$
\frac{d I_{\mathrm{HM}}}{d t}=M(\ldots)-\delta H\left(I_{\mathrm{HM}}\right)
$$

where $\delta$ is the infrastructure's depreciation rate and $H\left(I_{H M}\right)$ states functional relationship of public infrastructure and productivity of each RU. According to Muneepeerakul and Anderies (2017), many shared infrastructures can be modeled by threshold functions. Given that $H\left(I_{H M}\right)$ shows threshold behavior, they used a piecewise linear function to capture such behavior through Eq. A.2.

$$
H\left(I_{H M}\right)= \begin{cases}h, & I_{H M}>I_{m} \\ h\left(\frac{I_{H M}-I_{0}}{I_{m}-I_{0}}\right), & I_{0} \leq I_{H M} \leq I_{m} \\ 0, & I_{H M}<I_{0}\end{cases}
$$

where $h$ represents the maximum amount of harvest by each user under no restriction and $I_{0}$ and $I_{m}$ are the lower bound and upper bound thresholds of $I_{H M}$, respectively. Also, $M(.$.$) is the maintenance function (Eq. A.3) and depends on the$ social structure of the system.

$$
M(\ldots)=\mu y p C U N R H\left(I_{\mathrm{HM}}\right)
$$

In Eq. A.3, given the number of users $N, R U N H\left(I_{H M}\right)$ is the total harvest from the natural infrastructure. The RUs sell total harvest at price $p$ to generate revenue. Subsequently, they assign a proportion $C$ of revenue to PIP's for their contribution. Meanwhile, the PIP's spend proportion $y$ of $C$ on maintaining public infrastructure through the maintenance function $M(\ldots)$. Also, $\mu$ is the maintenance effectiveness of PIP's investment.

The second variable is the resource level, $R$. They assumed the dynamics of resource to be

$$
\frac{d R}{d t}=G(R)-U N R H\left(I_{\mathrm{HM}}\right)
$$

Natural infrastructure is assumed to invoke the conservation law comprising of 
regenerating capacity $(G(R)=g-d R)$ and total unit of harvest, $R U N H\left(I_{H M}\right)$. The definition presented for $G$ is the simplest model for natural infrastructure where $g$ and $d$ are the natural replenishment and the loss rates, respectively.

The strategic behavior of the resource users (RUs) is captured by employing a replicator equation. Indeed, replicator dynamics provide modelers with simple, realistic social mechanism where agents follow and replicate better-off strategies. The two possible strategies considered for RUs are staying inside the system with the associated payoff of $\pi_{U}=(1-C) p R H\left(I_{H M}\right)$ or leaving the system with the payoff of $\mathrm{w}=\mathrm{w}$. According to the replicator equation:

$$
\frac{d U}{d t}=r U(1-U)\left(\pi_{U}-w\right)
$$

The replicator equation represents the fraction of time that RUs assign to working inside system given $C$ and $y$. Like RUs, there is also two alternatives for PIPs, working inside the system or working for another CIS which leads to system failure. Meanwhile, $C$ and $y$ characterize the strategy or policy of PIPs. The PIPs will participate in this coupled system only when $\pi_{p}=(1-y) p C R U N H\left(I_{H M}\right) \geq w_{P}$. In other words, the PIPs maintain the system when they are better-off than working outside. This condition is termed the PIP Participation Constraint (PPC).

Based on the system of three differential equations (Eqs. A.1. A.4. A.5), the sustainable equilibria, i.e., long-term system outcomes that satisfy the stability condition and PPC, can be expressed as follows:

$$
\begin{aligned}
i_{H M}^{*} & =\frac{y C U^{*} N R^{*}}{g} H\left(I_{H M}^{*}\right) \\
R^{*} & =\frac{g}{d}\left(\frac{i_{H M}^{*}}{y C}\right) \\
U^{*} & =\frac{1-C}{y C} \phi_{1} i_{H M}^{*}
\end{aligned}
$$

where $i_{H M}^{*}=\frac{I_{H M}^{*} \delta}{\mu p g}$ (indicates dimensionless) and $\phi_{1}=\frac{p g}{w N}$, a dimensionless group representing the relative lucrativeness of the system, namely the ratio of potential income - with the entire resource flow turned into income - relative to outside wage. The results reported in this study are based on the following parameter values: $h=$ $0.0005 ; \delta=0.1 ; I_{0}=0.3 ; I_{m}=3 ; g=100 ; d=0.02 ; N=1000 ; r=0.15 ; p=10$; $w=1 ; w_{p}=100 ;$ and $=0.001$.

\section{Simplifying Eq. 1 through timescale separation argument}

We first used the timescale separation argument to simplify the model: the dynamics of $R$ and $U$ are significantly faster than that of $I_{\mathrm{HM}}$ as establishment and development of human-made infrastructures is a time-consuming process. This argument, namely $(d R / d t=d U / d t=0)$, leads to

$$
U R N H\left(I_{\mathrm{HM}}\right)=g-\frac{l w}{(1-C) p H\left(I_{\mathrm{HM}}\right)}
$$




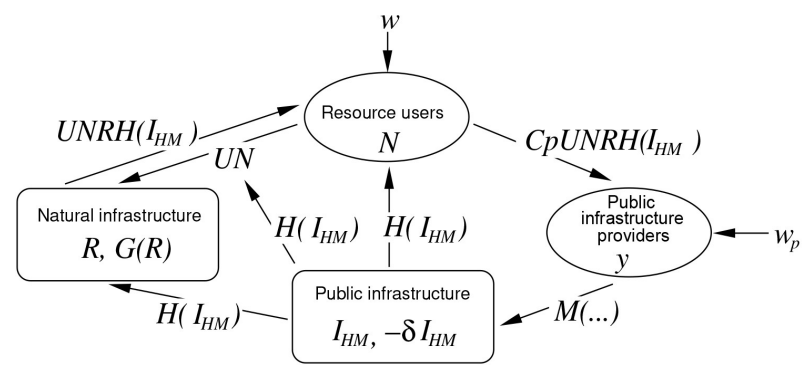

Figure A1. Schematic diagram of the dynamical system model. Taken from Muneepeerakul and Anderies (2017).

Applying such equality, the payoff associated with the PIPs, $\pi_{\mathrm{P}}=(1-$ y) $p C U N R H\left(I_{\mathrm{HM}}\right)$, can be written as:

$$
\pi_{\mathrm{P}}=(1-y) p C\left(g-\frac{l w}{(1-C) p H\left(I_{\mathrm{HM}}\right)}\right)
$$

Given the same argument, we derived $U$ as follows:

$$
U=\frac{(1-C) p}{N w}\left(g-\frac{l w}{(1-C) p H\left(I_{\mathrm{HM}}\right)}\right)
$$

\section{Non-dimensionlization}

Using some dimensionless groups, we rewrote the payoff associated with the PIPs as follows:

$$
\pi_{\mathrm{P}}=(1-y) \operatorname{Cpg}\left(1-\frac{\rho}{(1-C) \phi_{1} H(x)}\right)
$$

where, $x=\frac{I_{\mathrm{HM}}}{I_{\mathrm{m}}}$ is the rescaled $I_{\mathrm{HM}}$. Also, $\phi_{1}=\frac{p g}{w N}$ is defined as the potential income - with the entire resource flow turned into income - relative to outside wage and $\rho=\frac{l}{h N}$ indicates the relative natural loss rate of the resource compared to the maximum harvest rate. Accordingly, we can write the resilience-based objective function as follows:

$$
\begin{aligned}
& \left(x-x_{c}\right)\left(\frac{\pi_{\mathrm{P}}}{w_{\mathrm{P}}}-1\right)= \\
& \quad\left(x-x_{c}\right)\left((1-y) C \phi_{2}\left(1-\frac{\rho}{(1-C) \phi_{1} H(x)}\right)-1\right)
\end{aligned}
$$

where $\phi_{2}=\frac{p g}{w_{\mathrm{P}}}$ is a dimensionless group indicating potential income relative to PIP's alternative opportunities.

Likewise, we used the dimensionless groups to rewrite the Eq. A.8 as follows: 


$$
U=(1-C) \phi_{1}\left(1-\frac{\rho}{(1-C) \phi_{1} H(x)}\right)
$$

Using the above equation, we rewrote the performance-based objective function as:

$$
U+\frac{\pi_{P}}{N w}=(1-y C) \phi_{1}\left(1-\frac{\rho}{(1-C) \phi_{1} H(x)}\right)
$$

We also rewrote the Eq. 3 in dimensionless form (Eq. 4) in the main text using the above dimensionless groups. In this equation, $\theta_{\mathrm{m}}=\frac{\delta I_{\mathrm{m}}}{\mu p g}$ is the dimensionless group indicating decay rate of infrastructure at $I_{\mathrm{m}}$ relative to maximum maintenance.

\section{Deriving $\hat{y}_{\mathrm{R}}, \hat{y}_{\mathrm{P}-\mathrm{PIP}}$ and $\hat{y}_{\mathrm{P}-\mathrm{SYS}}$}

In order to derive the governance policies that maximize the objective functions associated with the resilience and the performances of the system represented by Eq. 1, we applied the HJB approach (Eqs.8, 9 and 10).

We then evaluated the first-order condition by taking the derivative of the terms inside the sqaure brackets on the right-hand side of the HJB equation (Eq. 8) with respect to the control variable, $y$ (i.e. the investment policy). The derivative,

$$
\left(1-\frac{\rho}{(1-C) \phi_{1} H(x)}\right)\left[\frac{d V}{d x} \frac{C}{\theta_{\mathrm{m}}}-C \phi_{2}\left(x-x_{c}\right)\right]
$$

reveals an on-and-off form of the policy associated with the resilience of the system: when the above expression is positive, we should invest all in the infrastructure maintenance, $y=1$; otherwise, we should not invest at all, $y=0$.

Likewise, evaluating the first-order condition for the Eqs. 9 and 10,

$$
\begin{aligned}
& \left(1-\frac{\rho}{(1-C) \phi_{1} H(x)}\right)\left[\frac{d V}{d x} \frac{C}{\theta_{\mathrm{m}}}-C \phi_{1}\right], \\
& \left(1-\frac{\rho}{(1-C) \phi_{1} H(x)}\right)\left[\frac{d V}{d x} \frac{C}{\theta_{\mathrm{m}}}-C \phi_{2}\right],
\end{aligned}
$$

also result in on-and-off policies in case of the performance priority.

In this study, the dimensionless groups associated with the base line scenario (corresponding to $g=100, w=1)$ are as follows: $\phi_{1}=1, \phi_{2}=10, \rho=0.2, \theta_{\mathrm{m}}=0.06$. For the purpose of the sensitivity analysis, we analyzed the model for different values of replenishing rates of resource, $g=\{90,100,110\}$, and per capita wage from working outside, $w=\{0.9,1,1.1\}$. 